\title{
Triangle Density and Contractibility
}

\author{
MATTHIAS KRIESELL \\ Instititut für Mathematik (A), Universität Hannover, Welfengarten 1, D-30167 Hannover, Germany \\ (e-mail: kriesell@math.uni-hannover.de)
}

Received 28 October 2002; revised 16 June 2003

\begin{abstract}
Let $G$ be a noncomplete $k$-connected graph such that the graphs obtained from contracting any edge in $G$ are not $k$-connected, and let $t(G)$ denote the number of triangles in $G$. Thomassen proved $t(G) \geqslant 1$, which was later improved by Mader to $t(G) \geqslant \frac{1}{3}|V(G)|$.

Here we show $t(G) \geqslant \frac{2}{3}|V(G)|$ (which is best possible in general).

Furthermore it is proved that, for $k \geqslant 4$, a $k$-connected graph without two disjoint triangles must contain an edge not contained in a triangle whose contraction yields a $k$-connected graph. As an application, for $k \geqslant 4$ every $k$-connected graph $G$ admits two disjoint induced cycles $C_{1}, C_{2}$ such that $G-V\left(C_{1}\right)$ and $G-V\left(C_{2}\right)$ are $(k-3)$-connected.
\end{abstract}

\section{Introduction}

All graphs considered here are taken to be finite, undirected, and simple. For terminology not defined here I would like to refer to [1] or [2].

An edge in a $k$-connected graph $G$ is called $k$-contractible if its contraction yields a $k$-connected graph. A noncomplete $k$-connected graph which has no $k$-contractible edge at all is called contraction critically $k$-connected.

For $k \leqslant 3$, there exists no contraction critically $k$-connected graph, whereas for each $k \geqslant 4$, there exist infinitely many nonisomorphic contraction critically $k$-connected graphs. The contraction critically 4-connected graphs are characterized to be the squares of cycles of length at least six plus the line graphs of cubic cyclically 4-edge-connected graphs. For various references to these results, see [4]. Every vertex in such a graph has degree four and is incident with at least two (edge-disjoint) triangles. Therefore, every contraction critically 4-connected graph has at least $\frac{2}{3}|V(G)|$ triangles, and this is attained for the linegraphs of (the infinitely many) noncomplete cubic cyclically 4-edge-connected graphs.

Here it will be proved that the number $t(G)$ of triangles in a contraction critically $k$-connected graph $G$ is at least $\frac{2}{3}|V(G)|$. This improves a result in [5], stating that $t(G) \geqslant \frac{1}{3}|V(G)|$, which in turn improves a result in [6], stating that $t(G) \geqslant 1$. As we have seen above, the constant $\frac{2}{3}$ is sharp in general, but probably not for increasing connectivity (see Section 3). 
Furthermore, the existence of large systems of edge-disjoint or vertex-disjoint triangles in contraction critically $k$-connected graphs is discussed. In [6], the result $t(G) \geqslant 1$ for every contraction critically $k$-connected graph has been used to prove that, for $k \geqslant 4$, every $k$-connected graph $G$ has an induced cycle $C$ such that $G-V(C)$ is $(k-3)$-connected. Here it will be shown that $G$ contains even two disjoint induced cycles $C_{1}, C_{2}$ such that $G-V\left(C_{1}\right)$ and $G-V\left(C_{2}\right)$ are $(k-3)$-connected. Here 'two' can not be improved in general.

\section{Large systems of triangles}

Let us recall some concepts of connectivity theory from [5]. For a graph $G$, let $\mathscr{T}(G):=$ $\{T \subseteq V(G): G-T$ disconnected and $|T|=\kappa(G)\}$ be the set of smallest separators of $G$, where $\kappa(G)=: k$ denotes the connectivity of $G$. For an arbitrary set $\mathscr{S}$ of subsets of $V(G)$, let $\mathscr{T}_{\mathscr{S}}(G):=\{T \in \mathscr{T}(G): S \subseteq T$ for some $S \in \mathscr{S}\}$. For $T \in \mathscr{T}_{\mathscr{S}}(G)$, any union of the vertex sets of at least one but not of all components of $G-T$ is called a $T-\mathscr{S}$-fragment. An $\mathscr{S}$-fragment is a $T-\mathscr{S}$-fragment for some $T \in \mathscr{T}_{\mathscr{S}}(G)$, and an $\mathscr{S}$-end is an inclusion minimal $\mathscr{S}$-fragment. If $\mathscr{S}=\emptyset$ then $\mathscr{T} \mathscr{S}(G)=\mathscr{T}(G)$, and in this case we omit $\mathscr{S}$ in the notation, thus defining $T$-fragments, fragments and ends. If $A$ is a $T-\mathscr{S}$-fragment in $G$ then so is $\bar{A}:=V(G)-(A \cup T)$. (At the risk of being somewhat imprecise, readability is probably increased by omitting a reference to $G$ in the notion of $\bar{A}$.) A fragment of cardinality 1 is called trivial. Note that if $F$ is a $T$-fragment in $G$ then $T=N_{G}(F)$, where $N_{G}(F):=\{y \in V(G)-F$ : there exists an $x \in F$ such that $x y \in E(G)\}$ denotes the neighbourhood of $F$ in $G$.

For two fragments $F_{1}, F_{2}$ of $G$, define

$$
T_{G}\left(F_{1}, F_{2}\right):=\left(F_{1} \cap N_{G}\left(F_{2}\right)\right) \cup\left(N_{G}\left(F_{1}\right) \cap N_{G}\left(F_{2}\right)\right) \cup\left(N_{G}\left(F_{1}\right) \cap F_{2}\right) .
$$

Clearly, $N_{G}\left(F_{1} \cap F_{2}\right) \subseteq T_{G}\left(F_{1}, F_{2}\right)$, and

$$
V(G)-\left(\left(F_{1} \cap F_{2}\right) \cup T_{G}\left(F_{1}, F_{2}\right)\right)=\overline{F_{1}} \cup \overline{F_{2}} \neq \emptyset .
$$

Therefore, if $F_{1} \cap F_{2} \neq \emptyset$ then

$$
\begin{aligned}
k \leqslant\left|N_{G}\left(F_{1} \cap F_{2}\right)\right| \leqslant\left|T_{G}\left(F_{1}, F_{2}\right)\right| & =\left|F_{1} \cap N_{G}\left(F_{2}\right)\right|+\left|N_{G}\left(F_{1}\right)-\overline{F_{2}}\right| \\
& =\left|F_{1} \cap N_{G}\left(F_{2}\right)\right|+k-\left|\overline{F_{2}} \cap N_{G}\left(F_{1}\right)\right| .
\end{aligned}
$$

From this inequality chain we obtain the following, fundamental property of fragments:

$$
\left.\begin{array}{l}
\text { For intersecting fragments } F_{1}, F_{2}, \\
\left|F_{1} \cap N_{G}\left(F_{2}\right)\right| \geqslant\left|\overline{F_{2}} \cap N_{G}\left(F_{1}\right)\right|, \\
\text { and if equality holds then } \\
F_{1} \cap F_{2} \text { is a } T_{G}\left(F_{1}, F_{2}\right) \text {-fragment. }
\end{array}\right\}
$$

The key observation in the proof of [5, Theorem 4], which states that $t(G) \geqslant \frac{1}{3}|V(G)|$ for every contraction critically $k$-connected graph $G$, is that whenever a vertex $x$ is not contained in a triangle in $G$ then there exists a 'small' nontrivial $T$-fragment $A$ such that $T$ contains $x$ and a neighbour of $x$. (In $A$, a neighbour of $x$ which is contained in 'many' triangles can be located - see [5, Theorem 3] - which then helps to prove the statement.) 
Here this is generalized by proving that even if $x$ is contained in at most one triangle in $G$ then there will be a 'small' nontrivial $T$-fragment such that $T$ contains $x$ and a neighbour of $x$.

We will need the following lemma, which follows more or less from the considerations in $[5]$.

Lemma 2.1. Let $G$ be a graph, let $\mathscr{S}$ be a set of subsets of $V(G)$, let $B$ be an $\mathscr{S}$-end, let $S \in \mathscr{S}$, and let $F$ be a $T$-fragment such that $T \cap B \neq \emptyset$ and $S \subseteq T-\bar{B}$.

Then one of $B, \bar{B}, F, \bar{F}$ has at most $\frac{\left|T-N_{G}(B)\right|}{2}$ vertices.

Proof. Let $T_{B}:=N_{G}(B)$. If $B \cap F \neq \emptyset$ and $\bar{B} \cap \bar{F} \neq \emptyset$ then $|B \cap T|=\left|\bar{F} \cap T_{B}\right|$ by (2.1) (applied to $B, F$ and $\bar{F}, \bar{B}$ for $\left.F_{1}, F_{2}\right)$. Again by (2.1), $B \cap F$ is a $T_{G}(B, F)$-fragment properly contained in $B$. Since $S \subseteq T_{G}(B, F), B \cap F$ is an $\mathscr{S}$-fragment properly contained in the $\mathscr{S}$-end $B$, which is absurd.

So either $B \cap F=\emptyset$ or $\bar{B} \cap \bar{F}=\emptyset$, and, symmetrically, either $B \cap \bar{F}=\emptyset$ or $\bar{B} \cap F=\emptyset$. It follows that $F \subseteq T_{B}$ or $\bar{F} \subseteq T_{B}$ or $B \subseteq T$ or $\bar{B} \subseteq T$. The property of $B$ being an $\mathscr{S}$ end will not be used furthermore, and $\left|T-T_{B}\right|=\left|T_{B}-T\right|$, so we may assume $F \subseteq T_{B}$ without loss of generality.

Let us assume that the assertion is not true. Then $\bar{F} \nsubseteq T_{B}$, and, without loss of generality, $B \cap \bar{F} \neq \emptyset$. By (2.1), $|B \cap T| \geqslant\left|F \cap T_{B}\right|=|F|>\left|T-T_{B}\right| / 2$, which implies $|\bar{B} \cap T|<\left|T-T_{B}\right| / 2$. So $\bar{B} \nsubseteq T$ neither, which implies $\bar{B} \cap \bar{F} \neq \emptyset$, and, by (2.1), $|\bar{B} \cap T| \geqslant$ $\left|F \cap T_{B}\right|=|F|>\left|T-T_{B}\right| / 2$, which is a contradiction.

It is not hard to see that an edge in a noncomplete $k$-connected graph is not $k$ contractible if and only if its endvertices are contained in some smallest separator of $G$ of cardinality $k$. This observation will be used throughout, without any further reference.

Lemma 2.2. Let $x$ be a vertex of degree at least 3 in a $k$-connected graph which is contained in at most one triangle such that every $k$-contractible edge incident with $x$ is contained in a triangle.

Then there exists a $T$-fragment $F$ such that the subgraph $G(F)$ induced by $F$ in $G$ is connected, $x \in T, N_{G}(x) \cap T \neq \emptyset$, and $2 \leqslant|F| \leqslant \frac{k-1}{2}$.

Proof. Set $\mathscr{S}:=\left\{\{x, y\}: y \in N_{G}(x) \wedge N_{G}(x) \cap N_{G}(y)=\emptyset\right\}$. By the conditions on $x, \mathscr{S}$ is not empty, and for every $S \in \mathscr{S}$, there exists a $T \in \mathscr{T}(G)$ such that $S \subseteq T$.

Suppose, to the contrary, that $G$ contains no connected $\mathscr{S}$-fragment $F$ such that $2 \leqslant|F| \leqslant \frac{k-1}{2}$. We may assume that $G$ contains no $\mathscr{S}$-fragment $F$ such that $2 \leqslant|F| \leqslant \frac{k-1}{2}$, for otherwise $G(F)$ would have at least two components, each of which must be trivial, so $x$ would be on at least two triangles.

Let $T^{\prime} \in \mathscr{T}_{\mathscr{S}}(G)$. Then there exists a $T^{\prime}$-fragment $F^{\prime}$ which does not intersect any triangle containing $x . F$ contains an $\mathscr{S}$-end $B$ having the same property. $B$ is intersected by some $S \in \mathscr{S}$, and there exists a $T \in \mathscr{T}(G)$ containing $S$. Let $F$ be a $T$-fragment. Since the vertex in $S-\{x\}$ has no neighbour in $N_{G}(x)$, neither $F$ nor $\bar{F}$ is trivial, and 
since $N_{G}(B)$ contains an $S^{\prime} \in \mathscr{S}$, neither $B$ nor $\bar{B}$ is trivial. By Lemma 2.1 , one of the $\mathscr{S}$-fragments $B, \bar{B}, F, \bar{F}$ has cardinality at most $\frac{k-1}{2}$, contradicting our assumption.

Lemma 2.2 is best possible in the sense that, for every $k \geqslant 3$ and every $b \geqslant 1$, there are infinitely many $k$-connected graphs $G$ which contain a vertex $x$ that is not incident with a $k$-contractible edge at all and is contained in exactly two triangles, but $|F| \geqslant b$ for every nontrivial $T$-fragment $F$ such $x \in T$ and $N_{G}(x) \cap T \neq \emptyset$ (so we can not replace 'at most one' with 'at most two' in the statement). An explicit construction is omitted to keep the paper short.

The existence of a fragment $F$ as in the conclusion of Lemma 2.2 implies $k \geqslant 5$, which has some consequences for the distribution of 4- or 3-contractible edges in 4- or 3-connected graphs, respectively. For example, independently from the characterization of the contraction critically 4-connected graphs mentioned above, it implies that every vertex in a contraction critically 4-connected graph is contained in two triangles.

Small but nontrivial fragments as provided by Lemma 2.2 will now help to partition contraction critically $k$-connected graphs into parts where the average number of triangles in which its vertices are contained is large. Let us introduce some further notation.

Let $G$ be a graph. For $x \in V(G)$, let $t_{G}(x):=\left|E\left(G\left(N_{G}(x)\right)\right)\right|$ denote the number of triangles incident with $x$. For $X \subseteq V(G)$, let $t_{G}(X):=\sum_{x \in X} t_{G}(x)$. If $X \neq \emptyset$ then $\overline{t_{G}}(X):=$ $\frac{t_{G}(X)}{|X|}$ is the average number of triangles in which a vertex in $X$ is contained. Consequently, any graph $G$ contains exactly $\frac{\overline{t_{G}}(V(G))}{3}|V(G)|$ triangles. For an integer $j \geqslant 0$, let $D_{G}^{j}:=$ $\left\{x \in V(G): t_{G}(x)=j\right\}$ be the set of vertices contained in exactly $j$ triangles in $G$, and let $D_{G}^{0,1}:=D_{G}^{0} \cup D_{G}^{1}$.

Lemma 2.3. Let $A$ be a nontrivial $T$-fragment of a graph of connectivity $k$ such that $G(A)$ is connected. Let $X \subseteq T \cap D_{G}^{0,1}$ be nonempty, and let $Y:=N_{G}(X) \cap A$. Then the following holds.

(1) If $|Y| \geqslant|X|$ then $\overline{t_{G}}(X \cup Y) \geqslant \frac{k-|A|}{2}$.

(2) If $3 \leqslant|A| \leqslant \frac{k-1}{2}$ then $\overline{t_{G}}(X \cup Y) \geqslant 2$.

(3) If $G(A)$ is a triangle and $T$ contains vertices $t_{1} \neq t_{2}$ such that $\left|N_{G}\left(t_{1}\right) \cap A\right|=$ $\left|N_{G}\left(t_{1}\right) \cap A\right|=1$ then $\overline{t_{G}}(X \cup Y) \geqslant \min \left\{k-3, \frac{6 k-15}{7}\right\}$.

Proof. For every edge $x y \in E(G(A))$,

$$
\left|N_{G}(x) \cap N_{G}(y)\right|=\left|N_{G}(x)\right|+\left|N_{G}(y)\right|-\left|N_{G}(x) \cup N_{G}(y)\right| \geqslant k+k-|T \cup A|=k-|A| .
$$

Since $G(A)$ is connected and $|A| \geqslant 2$, every vertex $x \in A$ has a neighbour in $A$, and thus $t_{G}(x) \geqslant k-|A|$.

Consequently, $t_{G}(Y) \geqslant|Y| \cdot(k-|A|)$ and if $|Y| \geqslant|X|$ then

$$
\overline{t_{G}}(X \cup Y) \geqslant \frac{|Y| \cdot(k-|A|)}{|X|+|Y|} \geqslant \frac{|Y| \cdot(k-|A|)}{2|Y|}=\frac{k-|A|}{2},
$$

as has been claimed in (1) of the statement. 
For the second part, suppose that $3 \leqslant|A| \leqslant \frac{k-1}{2}$. In particular, $k \geqslant 7$, and

$$
\frac{k-|A|}{2} \geqslant \frac{k+1}{4} \geqslant 2
$$

so (2) follows from (1) if $|Y| \geqslant|X|$.

Now let us assume, in addition, $|Y|<|X|$. It follows that $A=Y$, as all neighbours of $A-Y$ are contained in $(A \cap Y) \cup(T-X)$, which has fewer than $|T|=k$ vertices.

We now have to refine the argument from above. Set $X^{0}:=X \cap D_{G}^{0}$ and $X^{1}:=X \cap D_{G}^{1}$ (so $X$ is the disjoint union of $X^{0}$ and $X^{1}$ ).

Let $m:=\left|E_{G}(T, A)\right|$ denote the number of edges having one endvertex in $T$ and the other one in $A$. Since $|A| \geqslant 3$ and $G(A)$ is connected, we obtain $|E(G(A))| \geqslant 2$, so every $x \in X$ is nonadjacent to at least one vertex in $A$.

If $G(A)$ does not contain an independent set of $|A|-1$ vertices then every $x \in X^{0}$ is nonadjacent to at least two vertices in $A$. So $m \leqslant|A| \cdot k-2\left|X^{0}\right|-\left|X^{1}\right|$. On the other hand, every vertex in $A$ is adjacent to at least $k-(|A|-1)$ vertices in $T$, which implies $m \geqslant|A| \cdot k-|A| \cdot(|A|-1)$. It follows that $2\left|X^{0}\right|+\left|X^{1}\right| \leqslant|A| \cdot(|A|-1)$.

If $G(A)$ does contain an independent set of $|A|-1$ vertices then $G(A)$ is a star $K_{1,|A|-1}$. Let $c$ be the vertex of degree $|A|-1$ in $G(A)$. Then every $x \in X^{0} \cap N_{G}(c)$ has only one neighbour in $A$. There are, however, at most $|A|-1$ vertices in $X^{0}-N_{G}(c)$, which implies $m \leqslant$ $|A| \cdot k-2\left|X^{0}\right|+|A|-1-\left|X^{1}\right|$. Since $|E(G(A))|=|A|-1$, then $m \geqslant|A| \cdot k-2(|A|-1)$. It follows that $2\left|X^{0}\right|+\left|X^{1}\right| \leqslant 3 \cdot(|A|-1) \leqslant|A| \cdot(|A|-1)$.

Therefore, in either case we may estimate

$$
\begin{aligned}
\left|X^{1}\right|+|A| \cdot(k-|A|) & \geqslant\left|X^{1}\right|+|A| \cdot \frac{k+1}{2} \geqslant\left|X^{1}\right|+|A| \cdot(|A|+1) \\
& =\left|X^{1}\right|+|A| \cdot(|A|-1)+2|A| \geqslant\left|X^{1}\right|+2\left|X^{0}\right|+\left|X^{1}\right|+2|A| \\
& =2 \cdot(|X|+|A|),
\end{aligned}
$$

so

$$
\overline{t_{G}}(X \cup Y) \geqslant \frac{\left|X^{1}\right|+|A| \cdot(k-|A|)}{|X|+|A|} \geqslant 2 .
$$

These considerations imply (2) of the statement.

To prove (3), let $a_{i}$ be the vertex in $N_{G}\left(t_{i}\right) \cap A$, where $i \in\{1,2\}$. Note that $a_{1} \neq a_{2}$, for otherwise $\left|N_{G}\left(A-\left\{a_{1}\right\}\right)\right| \leqslant k-1$, which contradicts $k$-connectivity. Setting $H:=$ $G\left(A \cup T-\left\{t_{1}, t_{2}\right\}\right)$ we obtain, as above,

$$
\begin{gathered}
\left|N_{H}\left(a_{1}\right) \cap N_{H}\left(a_{2}\right)\right| \geqslant d_{H}\left(a_{1}\right)+d_{H}\left(a_{2}\right)-|V(H)| \geqslant(k-1)+(k-1)-(k+1)=k-3, \\
\text { and }\left|N_{H}\left(a_{i}\right) \cap N_{H}\left(a_{3}\right)\right| \geqslant(k-1)+k-(k+1)=k-2
\end{gathered}
$$

for $i \in\{1,2\}$. It follows that $t_{G}\left(a_{i}\right) \geqslant t_{H}\left(a_{i}\right) \geqslant(k-3)+(k-2)-1=2 k-6$ for $i \in\{1,2\}$, and $t_{G}\left(a_{3}\right) \geqslant t_{H}\left(a_{3}\right) \geqslant(k-2)+(k-2)-1=2 k-5$. If $|Y| \geqslant|X|$ then

$$
\overline{t_{G}}(X \cup Y) \geqslant \frac{|Y| \cdot(2 k-6)}{|X|+|Y|} \geqslant \frac{|Y| \cdot(2 k-6)}{2|Y|}=k-3 .
$$

If $|Y|<|X|$ then $Y=A$ as above. Set $S:=\left(N_{H}\left(a_{1}\right) \cap N_{H}\left(a_{2}\right)\right)-\left\{a_{3}\right\}$. As we have seen, $|S| \geqslant k-4$, and every vertex in $S$ is adjacent to $a_{3}$. So $S \cap X=\emptyset$, which implies $|X| \leqslant 4$, 
so $|X|=4$ and $t_{1}, t_{2} \in X$. Let $s_{1}, s_{2}$ be the vertices in $X-\left\{t_{1}, t_{2}\right\}$. Since they are in $X$ and adjacent to $a_{3}$, they are not adjacent to both $a_{1}, a_{2}$. Conversely, $a_{1}$ is adjacent to at least one of $s_{1}, s_{2}$, and so is $a_{2}$. So $s_{1}$ is adjacent to exactly one of $a_{1}, a_{2}$, and so is $s_{2}$, which implies $s_{1}, s_{2} \in X^{1}$. Now

$$
\overline{t_{G}}(X \cup Y) \geqslant \frac{2+(2 k-6)+(2 k-6)+(2 k-5)}{7}=\frac{6 k-15}{7}
$$

We need another, rather particular observation on contraction critically 5-connected graphs.

Lemma 2.4. Let $A$ be a $T$-fragment of cardinality 2 in a contraction critically 5-connected graph $G$ and let $t_{1} \neq t_{2}$ in $T$ such that $\left|N_{G}\left(t_{1}\right) \cap A\right|=\left|N_{G}\left(t_{2}\right) \cap A\right|=1$. Then one of $t_{1}, t_{2}$ has a neighbour of degree 5 in $T-\left\{t_{1}, t_{2}\right\}$.

Proof. Let us assume, to the contrary, that neither $t_{1}$ nor $t_{2}$ has a neighbour of degree 5 in $T-\left\{t_{1}, t_{2}\right\}$. Let $i \in\{1,2\}$, and let $a_{i}$ be the vertex in $N_{G}\left(t_{i}\right) \cap A$. Since $G$ is 5-connected, $a_{1} \neq a_{2}$. There exists a $T_{i} \in \mathscr{T}(G)$ containing $t_{i}$ and $a_{i}$. Then $a_{i}$ has neighbours in distinct components of $G-T_{i}$, which implies $a_{3-i} \in T_{i}$, as $N_{G}\left(a_{i}\right)-\left\{t_{1}\right\} \subseteq N_{G}\left(a_{3-i}\right) \cup\left\{a_{3-i}\right\}$.

By assumption, there exists no trivial $T_{i}$-fragment $F_{i}$, for otherwise the vertex contained in it had degree 5 and would be adjacent to $a_{1}, a_{2}$, and $t_{i}$, and would thus be contained in $T-\left\{t_{1}, t_{2}\right\}$. In particular, $\left|F_{i}\right| \geqslant 2$ and $\left|\bar{F}_{i}\right| \geqslant 2$.

Now take an arbitrary $T_{i}$-fragment $F_{i}$. By (2.1) it follows that $\left|F_{i} \cap T\right| \geqslant 2$ and $\left|\overline{F_{i}} \cap T\right| \geqslant$ 2, implying $\left|F_{i} \cap T\right|=\left|\overline{F_{i}} \cap T\right|=2$. Without loss of generality, $t_{2} \in F_{1}$ (otherwise we swap the roles of $F_{1}$ and $\overline{F_{1}}$ ), and $\left|F_{1} \cap F_{2} \cap T\right|=1$ (otherwise we swap the roles of $F_{2}$ and $\left.\overline{F_{2}}\right)$. Since $\overline{F_{1}} \cap \overline{F_{2}} \neq \emptyset, X:=F_{1} \cap F_{2}$ is a $T_{G}\left(F_{1}, F_{2}\right)$-fragment by (2.1). From $|X \cap T|=1$ and $A \subseteq T_{G}\left(F_{1}, F_{2}\right)$ it follows that $|X|=1$. But then the vertex in $X$ is a neighbour of $t_{2} \in T_{G}\left(F_{1}, F_{2}\right)$ of degree 5 in $T-\left\{t_{1}, t_{2}\right\}$, a contradiction.

Now we are equipped to prove the main result of this work.

Theorem 2.5. The vertex set of every contraction critically $k$-connected graph $G$ admits a partition $Z_{1}, \ldots, Z_{\ell}$ such that $\left|Z_{h}\right| \leqslant \max \left\{\frac{k-1}{2}, 3\right\}$ and $\overline{t_{G}}\left(Z_{h}\right) \geqslant 2$.

Proof. If $D:=D_{G}^{0,1}=\emptyset$ then, among others, the partition of $V(G)$ into sets of cardinality 1 has the properties of the assertion.

So we may assume that $D$ is not empty. By Lemma $2.2, k \geqslant 5$. Let us call a $T$-fragment $A$ good throughout this proof if either

(G1) $G(A)$ is connected, $2 \leqslant|A| \leqslant \frac{k-1}{2}$, and $\overline{t_{G}}\left(X \cup\left(N_{G}(X) \cap A\right)\right) \geqslant 2$ holds for every nonempty $X \subseteq T \cap D$, or

(G2) $G(A)$ is a triangle, and $T$ contains vertices $t_{1} \neq t_{2}$ such that $\left|N_{G}\left(t_{1}\right) \cap T\right|=$ $\left|N_{G}\left(t_{2}\right) \cap A\right|=1$.

Note that if $A$ is a good $T$-fragment then by means of (G2) we have $\overline{t_{G}}\left(X \cup\left(N_{G}(X) \cap A\right)\right) \geqslant$ 2, for every nonempty $X \subseteq T \cap D$, by the third part of Lemma 2.3. (So the option (G2) for a good fragment is designed only for the cases $k \in\{5,6\}$.) 
We construct, inductively, a sequence $\left(\alpha_{i}=\left(A_{i}, T_{i}, X_{i}, Y_{i}\right)\right)$ of length $\ell \geqslant 1$ as follows.

Let $i \geqslant 1$ and suppose that $\alpha_{h}$ has been constructed for $h \in\{1, \ldots, i-1\}$. If $D-$ $\bigcup_{h=1}^{i-1} X_{h}=\emptyset$ then the construction is finished, otherwise we proceed as follows.

Choice (A). If there exists a good $T$-fragment $A$ such that $X:=T \cap\left(D-\bigcup_{h=1}^{i-1} X_{h}\right)$ is not empty then we set $\alpha_{i}:=\left(A_{i}, T_{i}, X_{i}, Y_{i}\right):=\left(A, T, X, N_{G}(X) \cap A\right)$ and construct the next item.

Choice (B). Otherwise, we take any $x \in D-\bigcup_{h=1}^{i-1} X_{h}$. By Lemma 2.2 there exists a $T$-fragment $A$ such that $G(A)$ is connected, $2 \leqslant|A| \leqslant \frac{k-1}{2}, x \in T$, and $T \cap N_{G}(x) \neq \emptyset$. Since Choice (A) has not been applied here, $A$ is not good, and $|A|=2$ follows from Lemma 2.3. The set $X:=\left\{x \in D-\bigcup_{h=1}^{i-1} X_{h}: x \in T, T \cap N_{G}(x) \neq \emptyset\right\}$ is not empty, and we take $\alpha_{i}:=\left(A_{i}, T_{i}, X_{i}, Y_{i}\right):=\left(A, T, X, N_{G}(X) \cap A\right)$ and construct the next item.

Every vertex $a$ in some $A_{i}$ has a neighbour $b$ in $A_{i}$, and

$$
\left|N_{G}(a) \cap N_{G}(b)\right| \geqslant d_{G}(a)+d_{G}(b)-\left|T_{i} \cup A_{i}\right| \geqslant \frac{k-1}{2} \geqslant 2 .
$$

It follows that $\bigcup_{h=1}^{\ell} A_{h} \subseteq V(G)-D$. In particular, $\bigcup_{h=1}^{\ell} Y_{h} \subseteq V(G)-D$, and since $X_{i} \subseteq D$, $X_{i}$ is disjoint from every $A_{j}$ and every $Y_{j}$.

By construction, the $X_{j}$ are pairwise disjoint. The same holds for the $Y_{j}$, as we will see now. Let $i<j$ be two indices in $\{1, \ldots, \ell\}$.

Let us assume, to the contrary, that there exists an $a \in Y_{i} \cap Y_{j} \subseteq A_{i} \cap A_{j}$. Then $a$ must have a neighbour $x$ in $X_{j}$. Since $X_{j} \cap A_{i}=\emptyset, x \in T_{i}$ follows. Since

$$
x \in D-\bigcup_{h=1}^{j-1} X_{h} \subseteq D-\bigcup_{h=1}^{i-1} X_{h}
$$

but $x \notin X_{i}, \alpha_{i}$ has been chosen according to Choice (B). Consequently:

There is no good $T$-fragment $A$ such that $T \cap\left(D-\bigcup_{h=1}^{i-1} X_{h}\right)$ is not empty.

In particular, $A_{j}$ is not good (recall that $x \in T_{j} \cap\left(D-\bigcup_{h=1}^{i-1} X_{h}\right)$ ), so $\alpha_{j}$ has been chosen according to Choice (B), too. Since $x \in X_{j}-X_{i}, x$ has a neighbour in $T_{j}$ but no neighbour in $T_{i}$, implying that $T_{i} \neq T_{j}$. Therefore, $A_{i} \neq A_{j}$.

Let $A_{i}=\left\{a, b_{i}\right\}$ and $A_{j}=\left\{a, b_{j}\right\}$. Since $b_{i} \in T_{j}, b_{i}$ has a neighbour $t_{1}$ in $\overline{A_{j}}$. Clearly, $t_{1} \in T_{i}$ and $N_{G}(a)-\left\{b_{i}\right\}=T_{i}-\left\{t_{1}\right\}$, so $d_{G}(a)=k$. Symmetrically, there exists a $t_{2} \in$ $N_{G}\left(b_{j}\right) \cap \overline{A_{i}}$, and $N_{G}(a)-\left\{b_{j}\right\}=T_{j}-\left\{t_{2}\right\}$. It follows that $T_{i} \cap T_{j}=N_{G}(a)-\left\{b_{i}, b_{j}\right\}$, and $T:=N_{G}\left(A:=\left\{a, b_{i}, b_{j}\right\}\right)=\left(T_{i} \cap T_{j}\right) \cup\left\{t_{1}, t_{2}\right\}$. Since $|V(G)| \geqslant k+4, A$ is a $T$-fragment, and $\left|N_{G}\left(t_{1}\right) \cap A\right|=\left|N_{G}\left(t_{2}\right) \cap A\right|=1$. Recall that $b_{j} \in T_{i}$ is not adjacent to $x$, so $N_{G}\left(b_{j}\right)-$ $\{a\}=T_{j}-\{x\}$. In particular, $G(A)$ is a triangle.

Now $A$ is a good $T$-fragment and $x \in T \cap\left(D-\bigcup_{h=1}^{i-1} X_{h}\right)$, which violates (2.2).

This contradiction proves $Y_{i} \cap Y_{j}=\emptyset$.

We will now see that, unless we are in a rather particular situation, $\overline{t_{G}}\left(X_{i} \cup Y_{i}\right) \geqslant 2$ holds. So let us identify this setting.

If $X_{i}, Y_{i}$ have been chosen according to Choice (A) then $\overline{t_{G}}\left(X_{i} \cup Y_{i}\right) \geqslant 2$; this is trivially true if $A$ is good by means of (G1), and it follows from Lemma 2.3 otherwise. 
Otherwise, $\left|A_{i}\right|=2$, say $A_{i}=\left\{a_{1}, a_{2}\right\}$. Let $S:=N_{G}\left(a_{1}\right) \cap N_{G}\left(a_{2}\right)$, so $|S| \geqslant k-2$. If $x \in S$ has a neighbour in $T_{i}$ then $x$ is contained in at least two triangles, so $x \in T-X_{i}$. It follows that $\left|X_{i}\right| \leqslant 2$, and, consequently, $\left|Y_{i}\right| \geqslant\left|X_{i}\right|$. If $k \geqslant 6$ then $\overline{t_{G}}\left(X_{i} \cup Y_{i}\right) \geqslant \frac{k-\left|A_{i}\right|}{2} \geqslant 2$ by Lemma 2.3; so let us assume $k=5$ from now on.

If $t_{G}\left(a_{1}\right) \geqslant 4$ and $t_{G}\left(a_{2}\right) \geqslant 4$ then $\overline{t_{G}}\left(X_{i} \cup Y_{i}\right) \geqslant 2$, so we may assume that $t_{G}\left(a_{1}\right) \leqslant 3$ without loss of generality. In particular, $|S| \leqslant 3$, so $|S|=3=t_{G}\left(a_{1}\right)$, and there exist $t_{1} \neq t_{2}$ in $T_{i}$ such that $N_{G}\left(t_{1}\right) \cap A=\left\{a_{1}\right\}$ and $N_{G}\left(t_{2}\right) \cap A=\left\{a_{2}\right\}$. Furthermore, $S$ is independent in $G$. By Lemma 2.4, $t_{1}$ or $t_{2}$ has a neighbour in $S$, and since $t_{G}\left(a_{1}\right)=3, t_{2}$ must have a neighbour in $S$, which in turn implies $t_{G}\left(t_{2}\right) \geqslant 1$ and $t_{G}\left(a_{2}\right) \geqslant 4$. Now if $X_{i}$ would contain a vertex $x$ with $t_{G}(x) \geqslant 1$ then $\overline{t_{G}}\left(X_{i} \cup Y_{i}\right) \geqslant 2$. Hence we may assume $X_{i}=\left\{t_{1}\right\}$, which implies $Y_{i}=\left\{a_{1}\right\}$. By definition of $X_{i}, t_{1}$ must have a neighbour in $T$, which can only be $t_{2}$ since $t_{G}\left(t_{1}\right)=0$.

Let $j \neq i$. We claim that $A_{j}$ is disjoint from $A_{i}$.

Assume first that $\left|A_{j}\right|=2$. If $A_{j}=A_{i}$ then (since $A_{i}=A_{j}$ is not a good fragment) $X_{j} \subseteq\left\{t_{1}, t_{2}\right\}$; but then $t_{1}, t_{2} \in X_{\min \{i, j\}}$, which is absurd. So $A_{j} \neq A_{i}$.

Furthermore, $A_{j} \neq\left\{a_{1}, t_{1}\right\}$, since the latter set is adjacent to all 5 vertices in $A_{i} \cup T_{i}$ $\left\{a_{1}, t_{1}\right\}$ and to some further neighbour of $t_{1}$ in $\overline{A_{i}}$. Suppose that $A_{j}=\left\{a_{1}, x\right\}$ holds for some $x \in S$. Now the two vertices in $S-\{x\}$ are adjacent to $a_{1}$ but not adjacent to $x$, which is impossible. It follows that $a_{1} \notin A_{j}$ and, symmetrically, $a_{2} \notin A_{j}$.

So we may assume that $\left|A_{j}\right| \neq 2$, which implies that $A_{j}$ is a good fragment by means of (G2) (the actual vertices $t_{1}, t_{2}$ do not coincide with the respective ones in the definition of a good fragment). Since $t_{G}\left(t_{1}\right)=0, t_{1} \notin A_{j}$, and since $t_{1}$ can not be contained in the neighbourhood of a good fragment by choice, $a_{1}, t_{2} \notin A_{j}$. Suppose that $a_{2} \in A_{j}$. But then $A_{j}-\left\{a_{2}\right\} \subseteq S$, which is absurd since $G(S)$ has no edge.

So we have proved:

$$
\left.\begin{array}{l}
\text { Either } \overline{t_{G}}\left(X_{i} \cup Y_{i}\right) \geqslant 2 \text {, or: } \\
k=5,\left|X_{i}\right|=1,\left|A_{i}\right|=2 \text {, and } \\
A_{i} \text { is disjoint from } A_{j} \text { for all } j \neq i .
\end{array}\right\}
$$

Now we can construct a partition as follows. If $\overline{t_{G}}\left(X_{i} \cup Y_{i}\right) \geqslant 2$ then we set $Z_{i}:=X_{i} \cup Y_{i}$. Otherwise, we set $Z_{i}:=X_{i} \cup A_{i}$; in this case, $A_{i}$ is disjoint from every $A_{j}$ with $j \neq i$, and thus from every $Y_{j}, j \neq i$, too; furthermore, $\overline{t_{G}}\left(X_{i} \cup A_{i}\right) \geqslant \frac{2 \cdot(k-2)}{3} \geqslant 2$.

It follows that the $Z_{h}$ are pairwise disjoint and satisfy $\overline{t_{G}}\left(Z_{h}\right) \geqslant 2$. If $Z:=\bigcup_{h=1}^{\ell} Z_{h}=$ $V(G)$ then the assertion is proved. Otherwise, we add a partition $Z_{\ell+1}, \ldots, Z_{\ell+|V(G)-Z|}$ of $V(G)-Z$ into parts of cardinality 1 . Since $D \subseteq Z$, the assertion is proved as well.

Corollary 2.6. A contraction critically k-connected graph $G$ has at least $\frac{2}{3}|V(G)|$ triangles.

One could equally well ask for large systems of edge-disjoint or vertex-disjoint triangles in contraction critically $k$-connected graphs. The concept of minimally $k$-connected graphs is helpful in proving the following.

Corollary 2.7. For $k \geqslant 4$, a contraction critically $k$-connected graph $G$ has at least $\frac{2}{9 k-6}|V(G)|$ edge-disjoint triangles. 
Proof. Recall that a $k$-connected graph $G^{\prime}$ is minimally $k$-connected if $G^{\prime}-e$ is not $k$-connected for every $e \in E\left(G^{\prime}\right)$. Clearly, there exists a minimally $k$-connected spanning subgraph of $G$. Since $E\left(G^{\prime}\right) \subseteq E(G)$ and $\mathscr{T}\left(G^{\prime}\right) \supseteq \mathscr{T}(G), G^{\prime}$ is contraction critically $k$ connected, too, and it suffices to prove the assertion for $G^{\prime}$.

Consider an arbitrary edge $x y$ in $G^{\prime}$. Since $G^{\prime}-x y$ is not $k$-connected but $G^{\prime}$ is, $G^{\prime}-x y$ has a $T$-fragment $F$ such that $x \in F, y \in \bar{F}$, and $|T| \leqslant k-1$. In particular, $x, y$ have at most $k-1$ common neighbours in $G^{\prime}-x y$, so $x y$ is contained in at most $k-1$ triangles of $G^{\prime}$.

So for every triangle $\Delta$ of $G^{\prime}$, there are at most $3 k-3$ other triangles which are not edge-disjoint from $\Delta$. Consequently, if we take a maximal set $S$ of edge-disjoint triangles in $G^{\prime}$ then the total number of triangles is at most $(3 k-2)|S|$. Conversely, by Corollary 2.6, $G^{\prime}$ has at least $\frac{2}{3}\left|V\left(G^{\prime}\right)\right|$ triangles, so $|S| \geqslant \frac{2}{9 k-6}\left|V\left(G^{\prime}\right)\right|$, as has been asserted.

By a more careful analysis the constant at $k$ in the bound in Corollary 2.7 can be improved. I expect, however a much better bound (see Conjecture 3.3 below).

Finding a large system of vertex-disjoint triangles seems to be a harder task. We will see that small nontrivial fragments are useful in this context, too. The following lemma suggests that there could be a tradeoff between (disjoint) triangles and $k$-contractible edges.

Lemma 2.8. Let $A$ be a $T$-fragment of a $k$-connected graph such that $G(A)$ is connected, $2 \leqslant|A| \leqslant \frac{k-1}{2}$, and $|\bar{A}| \geqslant 2$. Then either $G$ has two disjoint triangles or there exists a $k$ contractible edge which is not contained in a triangle of $G$.

Proof. Since the statement of the conclusion does not depend on $A$, we may choose a $T$ fragment $A$ such that $G(A)$ is connected, $2 \leqslant|A| \leqslant \frac{k-1}{2}$, and $|\bar{A}| \geqslant 2$ under the additional condition that $|A|$ is as large as possible. Note that $k \geqslant 5$. Let us assume that $G$ does not have two disjoint triangles.

Every edge in $G(A)$ is contained in at least $k-|A| \geqslant \frac{k+1}{2} \geqslant|A|+1$ triangles of $G$. At least three of these must intersect $T$, and so $G(A)$ does not contain two independent edges (these would be on disjoint triangles). It follows that $G(A)$ is a triangle or a star $K_{1,|A|-1}$.

Let $x \in \bar{A}$. Then $x$ is not contained in a triangle (for such a triangle would be disjoint from one of the (at least) three triangles containing any prescribed edge in $G(A)$ and some vertex in $T$. Therefore, we may assume that there is no $k$-contractible edge incident with $x$. By Lemma 2.2, there exists a $T_{x}$-fragment $A_{x}$ such that $G\left(A_{x}\right)$ is connected, $A_{x}$ contains $x$ and a neighbour $y_{x}$ of $x, 2 \leqslant\left|A_{x}\right| \leqslant \frac{k-1}{2}$, and $\left|\overline{A_{x}}\right| \geqslant 2$. Let $a_{x} \in A$ be a neighbour of $x$, and let $b_{x} \in A_{x}$ be a neighbour of $a_{x}$. Note that $b_{x}$ is not adjacent to $x$, and $a_{x}$ is not adjacent $y_{x}$, for $x$ is not contained in a triangle. Furthermore, $A_{x}$ does not intersect $\bar{A}$, for otherwise there would be an edge in $G\left(A_{x}\right)$ intersecting $\bar{A}$ which would be contained in a triangle disjoint from one of the triangles containing any prescribed edge in $G(A)$.

Recall that $a_{x} b_{x}$ is contained in at least $k-\left|A_{x}\right| \geqslant 3$ triangles. If $G(A)$ is a triangle then at least one of them, say $\Delta$, intersects $G(A)$ in exactly one vertex, and the edge in $G(A)-V(\Delta)$ is contained in three triangles distinct from $G(A)$, one of which is disjoint from $\Delta$. Hence we may assume that $G(A)$ is a star $K_{1,|A|-1}$. 
Let us assume that $|A| \geqslant 3$, let $c$ be the vertex of degree $|A|-1$ in $G(A)$, and let $D:=G(A)-\{c\}$. Note that $d \in D$ is not contained in a triangle which does not contain $c$, for such a triangle would be disjoint from one of the triangles in which any edge in $G(A)-\{d\}$ would be contained. So $N_{G}(d) \cap T$ is independent for all $d \in D$. In particular, $G(T)$ does not contain a triangle. Since $a_{x} b_{x}$ is on at least $k-\left|A_{x}\right| \geqslant \frac{k+1}{2}>|A|$ triangles, none of which intersects $\bar{A}$, we obtain $a_{x} \in T$ and $b_{x} \in A$. If $b_{x} \in D$ then $N_{G}\left(b_{x}\right) \cap T$ would not be independent, so $b_{x}=c$. Now $a_{x} b_{x}$ is on at most $|D|$ triangles intersecting $D$, which implies that $a_{x}$ has at least

$$
\frac{k+1}{2}-|D|=\frac{k+1}{2}-|A|+1 \geqslant \frac{k+1}{2}-\frac{k-1}{2}+1=2
$$

neighbours in $G(T)$. It follows that $d$ is not adjacent to $a_{x}$ for all $d \in D$. This property uniquely determines $a_{x}$, independently from the particular choice of $x$ !

Take any neighbour $z$ of $a_{x}$ in $T$, and let $x^{\prime}$ be a neighbour of $z$ in $\bar{A}$. Since $a_{x}=a_{x^{\prime}}$, $a_{x}, z, x^{\prime}$ form a triangle intersecting $\bar{A}$, which is impossible.

Hence we have proved that $G(A)$ is a star $K_{1,1}$. By choice of $A,\left|A_{x}\right|=2$ for all $x$, too. Let $S:=\left\{t \in T: A \subseteq N_{G}(t)\right\}$.

If $A_{x} \subseteq T$ then $A \subseteq T_{x}$. Since no triangle intersects $\bar{A}$ and $\left|T_{x} \cap \bar{A}\right| \geqslant\left|A_{x} \cap T\right|=2$ by (2.1), it follows that $T_{x} \cap \bar{A}=\left\{x, y_{x}\right\}$, and $\left|T \cap T_{x}\right|=k-4 \geqslant 1$, and $a_{x}, b_{x} \in S$. Note that $x$ is the unique neighbour of $a_{x}$ in $\bar{A}$. Since $A \cup\left(T_{x} \cap T\right)$ contains a triangle $\Delta$ and $S \subseteq V(\Delta),|S|=3<k=5$ follows. Moreover, $E(G(T)) \neq \emptyset$.

If $A_{x} \nsubseteq T$ then $A_{x}$ intersects $A$. As in the proof of Theorem 2.5, $x$ is the unique neighbour of $a_{x}$ in $\bar{A}$. Since $y_{x}$ is not adjacent to $a_{x}, a_{x} \in S$ follows, and since $b_{x}$ is not adjacent to all vertices of $T$ (as above), $|S|<k$. Moreover, $E(G(T)) \neq \emptyset$.

In either case, $|S|<k$, and for every $x \in \bar{A}$ there exists an $a_{x} \in S$ such that $N_{G}\left(a_{x}\right) \cap \bar{A}=$ $\{x\}$, implying that $|\bar{A}| \leqslant|S|<k$. Since $E(G(T)) \neq \emptyset$ and no triangle intersects $\bar{A}$ and $|\bar{A}| \geqslant 2, G(\bar{A})$ contains an edge. However, this is contained in $k-|\bar{A}| \geqslant 1$ triangles, a contradiction.

Now we are prepared for two interesting conclusions on disjoint triangles in contraction critically $k$-connected graphs and on high connectivity keeping induced cycles in arbitrary graphs, respectively.

Theorem 2.9. Every contraction critically $k$-connected graph has two disjoint triangles.

Proof. Let $G$ be a contraction critically $k$-connected graph. If every vertex of $G$ is contained in two triangles then it is straightforward to check that $G$ has two disjoint triangles. Otherwise, we may apply Lemma 2.2 to some vertex contained in at most one triangle and find a fragment as it is supposed to exist in the statement of Lemma 2.8, which then implies the assertion.

In [6], the existence of a single triangle in a contraction critically $k$-connected graph has been used to prove that, in any $k$-connected graph, $k \geqslant 4$, there exists an induced cycle $C$ such that $G-V(C)$ is $(k-3)$-connected. The proof seems to be somehow robust against 
generalizations to larger systems of distinct, edge-disjoint, or vertex-disjoint systems of high connectivity keeping cycles, obtained from the corresponding triangle systems in the contraction critical case. In particular, there seems to be no direct way to derive the following from Theorem 2.9 .

Theorem 2.10. For $k \geqslant 4$, every $k$-connected graph $G \neq K_{5}$ has two disjoint induced cycles $C_{1}, C_{2}$ such that $G-V\left(C_{1}\right)$ and $G-V\left(C_{2}\right)$ are $(k-3)$-connected.

Proof. Let us call an induced cycle $C$ of $G$ a good cycle if $G-V(C)$ is $(k-3)$-connected and $\left|N_{G}(x) \cap V(C)\right| \leqslant 3$ for every $x \in V(G)-V(C)$. As in the proof of the theorem in [6], we prove the stronger statement that $G$ contains two disjoint good cycles $C_{1}, C_{2}$ by induction on $|V(G)|$.

If the graph $G$ in question has two disjoint triangles then they will serve for $C_{1}, C_{2}$. Otherwise, we find a vertex which is contained in at most one triangle and may apply Lemma 2.2 and Lemma 2.8 as in the preceding proof to show that $G$ has a $k$-contractible edge $e=x y$ which is not contained in a triangle. By induction, the graph $G^{\prime}:=G / e$ obtained from contracting $e$ in $G$ to a single vertex $z$ has two disjoint good cycles $C_{1}^{\prime}, C_{2}^{\prime}$. Let $C_{i}$ be the subgraph induced in $G$ by $V\left(C_{i}^{\prime}\right)-\{z\}$ plus all vertices $w \in\{x, y\}$ such that there is an edge $a z$ in $C_{i}^{\prime}$ corresponding to an edge $a w$ in $G$. Since $e$ is not contained in a triangle, $C_{1}$ and $C_{2}$ remain disjoint induced cycles.

Take $i \in\{1,2\}$. If $x, y \notin V\left(C_{i}\right)$ then $z \notin V\left(C_{i}^{\prime}\right)$, so $C_{i}^{\prime}=C_{i}$, and $\left|N_{G}(x) \cap V\left(C_{i}\right)\right| \leqslant$ $\left|N_{G^{\prime}}(z) \cap V\left(C_{i}^{\prime}\right)\right| \leqslant 3$. Symmetrically, $\left|N_{G}(y) \cap V\left(C_{i}\right)\right| \leqslant 3$, so $\left|N_{G}(w) \cap V\left(C_{i}\right)\right| \leqslant 3$ for all $w \in V(G)-V\left(C_{i}\right)$. Since $x, y$ both have degree at least $k-3$ in $G-V\left(C_{i}\right), G-V\left(C_{i}\right)$ is $k$-connected. Hence $C_{i}$ is a good cycle in this case.

If $x, y \in V\left(C_{i}\right)$ then $G-V\left(C_{i}\right)=G^{\prime}-V\left(C_{i}^{\prime}\right)$ is $(k-3)$-connected. Since $x y$ is not contained in a triangle, $\left|N_{G}(w) \cap V\left(C_{i}\right)\right|=\left|N_{G^{\prime}}(w) \cap V\left(C_{i}^{\prime}\right)\right| \leqslant 3$ for all $w \in V(G)-V\left(C_{i}\right)$, so $C_{i}$ is a good cycle.

Finally, if $x \in V\left(C_{i}\right)$ and $y \notin V\left(C_{i}\right)$ then $\left|N_{G}(y) \cap V\left(C_{i}\right)\right|=1$ since $x y$ is not on a triangle and $C_{i}^{\prime}$ is an induced cycle in $G^{\prime} . G-V\left(C_{i}\right)$ is $k-3$-connected, since it is obtained from the $(k-3)$-connected graph $G^{\prime}-V\left(C_{i}^{\prime}\right)$ by adding the vertex $y$ and making it adjacent to at least $k-1$ vertices. We deduce $\left|N_{G}(w) \cap V\left(C_{i}\right)\right| \leqslant\left|N_{G^{\prime}}(w) \cap V\left(C_{i}^{\prime}\right)\right| \leqslant 3$ for every $w \in(V(G)-\{y\})-V\left(C_{i}\right)$ too, so $C_{i}$ is a good cycle. The same conclusion holds if $y \in V\left(C_{i}\right)$ and $x \notin V\left(C_{i}\right)$.

So $C_{1}, C_{2}$ are disjoint good cycles in $G$, and the induction works.

Theorem 2.10 generalizes the theorem in [6]. If it is possible to prescribe an edge in a high connectivity keeping cycle then this would give an affirmative answer to a conjecture of Lovász ( $c f$. [3]). Not even prescribing a single vertex in a high connectivity keeping induced cycle seems to be possible today, but forbidding a single vertex is possible, as an immediate consequence of Theorem 2.10 (or, with a worse loss of possibly 4 in connectivity, as an immediate consequence of the theorem in [6]).

Corollary 2.11. For $k \geqslant 4$ and for every vertex of a $k$-connected graph $G$ there exists an induced cycle $C$ not containing $x$ such that $G-V(C)$ is $(k-3)$-connected. 


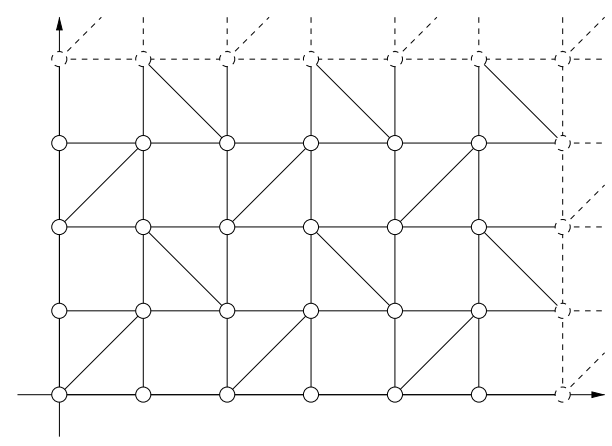

Figure 1.

\section{Problems and open questions}

Of course the question on the quality of the bound in Corollary 2.6 arises. In [4], for each $k \geqslant 4$ infinitely many contraction critically $k$-connected graphs $G$ with $t(G)=\frac{k}{6} \cdot|V(G)|$ if $k$ is even and $t(G)=\frac{k+3}{6} \cdot|V(G)|$ if $k$ is odd have been constructed. For odd $k$, a further improvement is possible.

Let $m, n \geqslant 4$ even integers. Define a graph $G_{m, n}$ by $V\left(G_{m, k}\right):=Z_{m} \times Z_{n}$ and

$$
\begin{aligned}
E\left(G_{m, n}\right)= & \left\{(x, y)(x+1, y),(x, y)(x, y+1):(x, y) \in V\left(G_{k}\right)\right\} \\
& \cup\left\{(x, y)(x+1, y+1),(x+1, y+2)(x+2, y+1): x \in Z_{m} \text { even and } y \in Z_{n} \text { even }\right\} .
\end{aligned}
$$

Figure 1 shows $G_{6,4}$ on the torus.

It is easy to see that $G_{m, n}$ is a toroidal 5-connected vertex-transitive graph where every edge is contained in some triangle and every vertex is on precisely 3 triangles. So $G$ has $|V(G)|$ triangles. Now, for odd $k \geqslant 7$, the graph

$$
G_{m, n, k}:=G_{m, n} \times \overbrace{K_{3} \times \cdots \times K_{3}}^{(k-5) / 2 \text { times }}
$$

is a vertex-transitive contraction critically $k$-connected $k$-regular graph where every vertex is on $\frac{k+1}{2}$ triangles. So $t\left(G_{m, n, k}\right)=\frac{k+1}{6} \cdot|V(G)|$.

I should guess that this is best possible.

Conjecture 3.1. For even $k$, every contraction critically $k$-connected graph $G$ has at least $\frac{k}{6} \cdot|V(G)|$ triangles, and for odd $k$, every contraction critically $k$-connected graph $G$ has at least $\frac{k+1}{6} \cdot|V(G)|$ triangles.

If $G$ is a contraction critically $k$-connected graph such that

$$
\{T \in \mathscr{T}(G): E(G(T)) \neq \emptyset\} \subseteq\left\{N_{G}(z): z \in V(G)\right\}
$$

then the statement of Conjecture 3.1 holds (and is best possible). To see this, take any vertex $x$. For every $y \in N_{G}(x)$, take a $T \in \mathscr{T}(G)$ containing $x, y$. Then $T=N_{G}(z)$ for some $z$. It follows that the minimum degree of $G\left(N_{G}(x)\right)$ is at least 1 , implying that 
$\left|E\left(G\left(N_{G}(x)\right)\right)\right| \geqslant\left\lfloor\frac{k+1}{2}\right\rfloor$, so every vertex is contained in at least $\left\lfloor\frac{k+1}{2}\right\rfloor$ triangles, which then proves $t(G) \geqslant \frac{k}{6} \cdot|V(G)|$ if $k$ is even and $t(G) \geqslant \frac{k+1}{6} \cdot|V(G)|$ if $k$ is odd.

From Conjecture 3.1 it would follow that the triangle density $t(G) / V(G)$ of contraction critically $k$-connected graphs $G$ grows linearly in $k$.

Conjecture 3.2. There exists a positive real $c$ such that, for every $k$, every contraction critically $k$-connected graph has at least $c \cdot k \cdot|V(G)|$ triangles.

It should be a hard task to prove that, 'next to' every $x \in D_{G}^{2}$ there is some suitable subset $Y(x)$ such that $\overline{t_{G}}(Y(x))$ is large enough and such that the union $\bigcup_{x \in D_{G}^{2}} Y(x)$ is also large enough compared with $D_{G}^{2}$. So a straightforward generalization of my proof of Theorem 2.5 towards Conjecture 3.2 is impossible.

Conjecture $3.2 \mathrm{had}$, if true, the following interesting consequence. (This can be shown using the same argument as in Corollary 2.7.)

Conjecture 3.3. There exists a positive real $c$ such that, for every $k$, every contraction critically k-connected graph has a system of at least $c \cdot|V(G)|$ edge-disjoint triangles.

It seems likely that Conjecture 3.3 can even be sharpened to vertex-disjoint triangle systems instead of edge-disjoint ones. A further generalization to vertex-disjoint induced high-connectivity keeping cycles as in Theorem 2.10 is not possible, not even if $c$ may depend on $k$, as is shown by the graphs obtained from cycles by adding $k-2$ new vertices and adding all edges from the new to the old ones: these graphs do not admit systems of more than $\min \{1, k-2\}$ vertex-disjoint cycles at all.

In [5], examples of contraction critically $k$-connected graphs $G$ have been constructed which contain a vertex $x$ with $t_{G}(x)=0$. In the construction, $k \geqslant 9$. On the other hand, $t_{G} \geqslant 2$ for every contraction critically 4-connected graph $G$. Most of the additional considerations in the proof of Theorem 2.5 concerning the case $k=5$ would be obsolete if $t_{G} \geqslant 1$ holds for every contraction critically 5-connected graph $G$; so far I do not know of a counterexample to this statement.

Problem 3.4. Is every vertex of a contraction critically 5-connected graph contained in a triangle?

It is an open problem whether there exist numbers $b_{5}$ and $h_{5}$ such that every 5connected graph $G$ with $|V(G)| \geqslant b_{5}$ can be contracted to a 5-connected graph $H$ such that $0<|V(G)|-|V(H)|<h_{5}$. Of course, the similar question can be formulated for arbitrary $k$ instead of 5 , but, tantalizingly, for $k<5$ the answer to that question is 'yes', and for $k>5$ it is 'no' [4]. So, with respect to this problem, it is of particular interest to understand contraction critically 5-connected graphs.

Again, let $x$ be a vertex of a contraction critically $k$-connected graph $G$. By Lemma 2.2 (or the results in [5]), there exists a $T$-fragment $A$ such that $x \in T, T \cap N_{G}(x) \neq \emptyset$, and $|A| \leqslant \frac{k-1}{2}$. From this it follows easily that $x$ is contained in a cycle of length at most 5 . It need not be contained in a triangle, and here is a gap to fill. 
Problem 3.5. Is every vertex of a contraction critically $k$-connected graph contained in a cycle of length 3 or 4 ?

Apparently, the existence of a short cycle containing some prescribed vertex in a contraction critically $k$-connected graph can not be used to find a high connectivity keeping induced cycle containing some prescribed vertex in an arbitrary $k$-connected graph. To adapt the proof technique of [6] it would be necessary that, if a vertex $x$ of a $k$-connected graph $G$ is not on some ('good') short cycle then there exists a contractible edge in $G$ not incident with $x$. Such a statement is not true in general: it is possible to construct $k$-regular $k$-connected line graphs where every clique has size $\lfloor k / 2\rfloor+1$ or $\lceil k / 2\rceil+1$ such that the shortest induced cycle of length exceeding three is arbitrarily large. If these graphs are large enough, then they must contain a set of $k$ vertices which are mutually far apart from each other, and so making a new vertex $x$ adjacent to all of these $k$ will produce a $k$-connected graph where $x$ is not contained in some short cycle. If $k \geqslant 6$ then, by the clique size condition, the endvertices of every edge not incident with $x$ have a common neighbour of degree $k$. So the edges not incident with $x$ are not $k$-contractible. So the following remains open here.

Problem 3.6. Is there a number $h$ such that for every $k>h$ and an arbitrary vertex $x$ of a k-connected graph $G$ there exists an induced cycle $C$ containing $x$ such that $G-V(C)$ is $(k-h)$-connected?

\section{References}

[1] Bondy, J. A. and Murty, U. S. R. (1976) Graph Theory with Applications, Macmillan.

[2] Diestel, R. (1997) Graph Theory, Vol. 173 of Graduate Texts in Mathematics, Springer.

[3] Kriesell, M. (2001) Induced paths in 5-connected graphs. J. Graph Theory 36 52-58.

[4] Kriesell, M. (2002) A survey on contractible edges in graphs of a given vertex connectivity. Graphs and Combinatorics 18 1-30.

[5] Mader, W. (1988) Generalizations of critical connectivity of graphs. Discrete Math. 72 267-283.

[6] Thomassen, C. (1981) Nonseparating cycles in k-connected graphs. J. Graph Theory 5 351-354. 